Article

\title{
Acupuncture on Lactate, Perceived Exertion in Recreational Athletes Submitted to HIIT
}

Carlos Soares Pernambuco ${ }^{1,2}$, Rodrigo Gomes de Souza Vale ${ }^{1,2}$, João Rafael Valentim-Silva ${ }^{4}$, Carlos Alberto de Souza Filho; Vinicius do Espirito Santo de Jesus'; Eliane da Silva Borges; Leandra Silva Cardoso4; Isabel Cristina Regazzi ${ }^{6}$, Estelio Henrique Martin Dantas 7

1- Estácio de Sá University, Cabo Frio Campus - Rio de Janeiro - Brazil rodrigovale@globo.com;

2- Exercise Physiology Laboratory - Lafiex/UNESA -vincius.jesus@estacio.br;

carlosalberto sf@outlook.com

3- North Paraná University - Unopar, Londrina, Paraná state - leandra.faria@hotmail.com

4- Lecturer at UNINORTE University Center, Rio Branco, Acre; Post-

Doctoral Fellow at the Nanobiotechnology Laboratory, Federal University of Acre, Rio

Branco, AC, Brazil - p.jrvalentim-silva@gmail.com

5- Laboratory of Bioscience of Human Motricity - Labimh/Unirio elianesborges@hotmail.com

6- Federal Fluminense University - Campus Rio das Ostras - RJ - violetaflora@hotmail.com

7- Tiradentes University - Sergipe- Brazil-esteliodantas@gmail.com

Corresponding author: karlos.pernambuco@hotmail.com

\begin{abstract}
Studies have demonstrated the positive effects of acupuncture on athletic performance. The aim of this study was to determine the acute effects of acupuncture on heart rate, the perceived exertion scale and lactate levels in recreational athletes. Fifteen competitive males engaged in HIIT. The characteristic was $29.86 \pm 2.51$ years old, heart rate reserve $59.00 \pm 3.33$, lactate $3.17 \pm 0.50 \mathrm{mM} / \mathrm{DL}$. The subjects were submitted to two exercise sessions. Both training sessions consisted of 10 burpees, 12 thrusters and 14 box jumps (75 $\mathrm{cm}$ high) for 12 minutes. Activity intensity was between 85 and $95 \%$ of maximum heart rate. Acupuncture points: ST36, L3, LI1 1. The student's t-test was adopted, Shapiro-Wilk test was applied for normality, and Pearson correlation. There was a positive correlation of $\mathrm{r}=0.69$

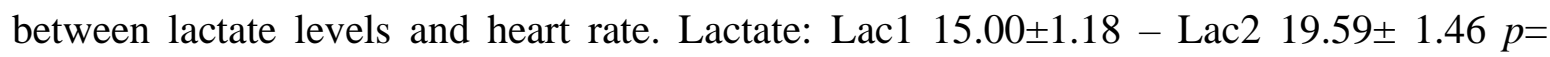
0.0001*; Heart 1rate: HRF 163.71 \pm 7.27 - HRF2 177.60 $\pm 6.99 p=0.0001 *$; Blood pressure: SBP1 174.86 $\pm 1.57-$ SBP2 180.86 $\pm 1.77 p=0.0001 *$; PES1: 19.4 \pm 1.14 ; PES2 16.8 $\pm 0.84 p=$ $0.0001 *$; weight 1 - 182,57 $\pm 12,05$; weight $2206,43 \pm 11,39 \mathrm{p}=0.0325^{*}$. Acupuncture increased lactate accumulation, heart rate and blood pressure, suggesting that the exertion reached after acupuncture is higher than without acupuncture. The acupuncture technics improved the athlete performance.
\end{abstract}

Keywords: Acupuncture, lactate, heart rate, physical performance, sustainability 


\section{Introduction}

The level of resting serum lactate is a potential predictor of muscle exertion during training or competition $(1,2)$. In this respect, high-intensity activities, like nearly all sports competitions, accumulate lactate due to cellular energy metabolism, which is unable to mobilize the entire amount of pyruvate produced for the Krebs cycle. As such, lactate dehydrogenase ( $\mathrm{LDH})$ action is required to convert this metabolite into lactate for export into the blood, given that the accumulation of one or the other inside the cell compromises adequate cell metabolism (3).

A series of actions were suggested to accelerate blood lactate clearance, such as active rest, consisting of low-intensity cyclic physical activity (4) and cold water immersion (5). This is because faster removal may be a physiological and athletic advantage, given that metabolically, lactate may decrease an athlete's physical performance, particularly when they compete in more than one event over a short period of time (6).

Acupuncture, an oriental technique with proven benefits for human health (7), involves needle insertion into specific sites of the body to stimulate them and release so-called chakra energy. According to traditional oriental medicine, vital energy retained at these points may be the cause of disorders (8).

Studies have demonstrated the positive effects of acupuncture on athletic performance. It has shown curative properties for joint and muscle injuries, in addition to improving sport performance, endurance, resistance, muscle strength and power (9-12).Specifically with respect to endurance, acupuncture causes positive changes in anaerobic threshold, maximum heart rate and faster post-exercise recovery $(10,13)$. However, its relationship with energy metabolism and lactate clearance has been little discussed in the literature, suggesting the need for investigations that may elucidate this possible relation.

Introduce techniques with low cost and low adverse effects promoted by non-steroidal anti-inflammatory drugs, like gastrointestinal bleeding, oliguria, fluid retention, decrease excretion of sodium (14). It is also essential to evaluate the suitability and sustainability of an intervention. Suitability refers to 'the quality of being right or appropriate for a particular person, purpose or situation' and Sustainability is 'the ability to be maintained at a certain rate or level'. It 'requires its own evaluation, apart from and usually after, an evaluation has shown positive results for the programmed intervention itself (15).

Thus, techniques that can improve athletic performance, promote tissue protection, and avoid injury without the use of medication should be explored by the sport community. Techniques such as acupuncture may be a viable strategy for increasing performance. Therefore, the aim of this study was to determine the acute effects of acupuncture on heart rate, the perceived exertion scale and lactate levels in recreational athletes engaged in high-intensity interval training.

\section{Materials and Methods}

This is a quasi-experimental study composed of a convenience sample of volunteers, without a control group (16). The study was approved by the UNESA (Estacio de Sá University) Research Ethics Committee, under protocol no. 3.203.302 and complied with Declaration of Helsinki guidelines. 
The sample consisted of 15 recreational athletes male Cross fitters engaged in high-intensity interval training, not using androgenic anabolic steroids, active for more than six months, with a minimum of five sessions per week, each lasting at least 50 minutes. Exclusion criteria were individuals with recent bone, muscle or joint injury, those in recovery, using analgesics or anti-inflammatories, orthoses or prostheses, alcohol drinkers and smokers, and those taking antidepressants, beta blockers, appetite suppressants or androgenic anabolic steroids. Fifteen of the 24 volunteers interviewed were selected for the study. (table 1)

Data collection occurred at the Exercise Physiology Laboratory of Estácio de Sá University, Cabo Frio Campus, with subjects sitting for 10 minutes before procedures. Blood pressure was measured with an aneroid sphygmomanometer (Premium Grafite, Brazil) and a stethoscope (Littmann Classic III, USA). To measure serum lactate, one drop of peripheral blood was collected from the ear lobe with a metal lancet and a test strip (BM Lactate REF- 03012654370 Accutrend PLUS), and analysis conducted with a lactate meter (Accutrend Lactate Roche, Manheim, Germany).

Height was measured with a stadiometer (Cardiomed - Brazil) accurate to 5 millimeters, and body mass index with a digital balance (Wiso, China). Blood was collected again 5 minutes after the intervention. The seven skin protocol for men was used to identify body fat percentage (17)

The subjects were submitted to two exercise sessions with a seven-day interval between them. Both training sessions consisted of 10 burpees, 12 thrusters at $85 \%$ maximum load and 14 box jumps ( $75 \mathrm{~cm}$ high) for 12 minutes. Activity intensity was between 85 and $95 \%$ of maximum heart rate, monitored by a Polar A370 (Finland). The participants were instructed to perform the task as fast as possible, with the largest number of repetitions, at the highest intensity possible during the entire intervention. They were verbally encouraged throughout the intervention and allowed a 30-second rest every 3 minutes (18-20).

Although the second session used the same methodological procedures, acupuncture was applied for 20 minutes before its onset, at points E36, IG4, IG11 and F3. Lactate was measured five minutes before and five minutes after the session.

It was applied a heterodox model acupuncture, puncturing the needle right perpendicular to skin. Points used were: stomach (ST) 36 is located $1 \mathrm{~cm}$ laterally to the tibial crest at the height of the groove below the tibial plateau; liver (L) 3 between the first and second metatarsal bones; IG4 at the angle between the first and second metacarpal; and large intestine (LI)11 at the lateral extremity of the elbow flexion skin crease (Lima, 2015).DongBang needles (China), caliber $0.20 \times 0.30 \mathrm{~mm}$, were used at an insertion depth of 1.00 to 3.00 centimeters. Needle application time was 20 minutes. The data were analyzed by IBM SPSS 23 software and presented as mean and standard deviation. The Shapiro-Wilk test was used to verify the normality and homogeneity of data variance. The student's t-test was adopted with a 5\% significance level. Pearson's correlation was conducted between lactate levels and heart rate.

\section{Results.}


After calls twenty four volunteers were interviewed but fifteen volunteers were accepted to participate the study. The others were refused: four by less time of practice, two by recent muscular lesions and three by steroids ingestion.

Table 1: Clinical and physical characteristics of the sample.

\begin{tabular}{|c|c|}
\hline Participant Characterization & $(n=15)$ \\
\hline Age (Years) & $29.86 \pm 2.51$ \\
\hline Weight (Kg) & $73.08 \pm 8.54$ \\
\hline Height (Cm) & $1.76 \pm 8.74$ \\
\hline \%Fat (7 skin) & $15.13 \pm 0.67$ \\
\hline BMI $\left(\mathrm{Kg} / \mathbf{m}^{2}\right)$ & $25.56 \pm 2.79$ \\
\hline Lactate (mM/DL) & $3.17 \pm 0.50$ \\
\hline HRR (BPM) & $59.00 \pm 3.33$ \\
\hline $\mathrm{SBP}(\mathrm{mm} / \mathrm{Hg})$ & $110.62 \pm 2.05$ \\
\hline DBP (mm/Hg) & $80.41 \pm 1.22$ \\
\hline
\end{tabular}

Legend: $\mathrm{Kg}=$ kilograms; $\mathrm{Cm}=$ centimeters; $\mathrm{BMI}=$ body mass index; $\mathrm{Kg} / \mathrm{m}^{2}=$ kilograms per square meter; $\%$ Body Fat $=$ seven skin, HRR $=$ heart rate reserve, $\mathrm{SBP}=$ systolic blood pressure, $\mathrm{DBP}=$ diastolic blood pressure.

The sample showed was characterized overweight by BMI $(25.56 \pm 2.79)$ and \% fat $(15.13 \pm 0.67)$ showed the group have lower fat mass, levels of lactate on rest $(3.17 \pm 0.50)$, may be all volunteer made a section of work out a day before

It was applied a Pearson correlation test between lactate levels and heart rate. There was a positive correlation of $\mathrm{r}=0.7846(p<0.05)$. Lactate showed greater accumulation after acupuncture (Fig. 1A) (before start test lac 3.17 \pm 0.50 ; lac1 19.59 \pm 1.46 and lac2 19.59 \pm 1.46 ; $p=0.0004)$. For HRF, acupuncture resulted in greater lactate accumulation after acupuncture (Fig. 1B) (HRF 59.71 \pm 4.10 , HR1 163.71 \pm 7.27 and HR2 177.60 $\pm 6.99 ; p=0.0001$ ). Blood pressure was also higher after acupuncture (SBP 110.32 \pm 3.10, SBP1 174.86 \pm 1.57 and SBP3 $180.86 \pm 1.77, p=0.0001$ ), the consumption of oxygen was VO2 1the perceived exertion scale (PES) was lower in the group after acupuncture (PES1 - 19.4 \pm 1.14 and PES2 - 16.8 \pm 0.84 ; $p=0.001$ ). Finally, the weight reached at final of the test was WEI1 $-40.00 \pm 7.07$, WEI $2-$ $49.00 \pm 5.48$ (Watts). 
Figure 1: Statistical results of variables analyzed

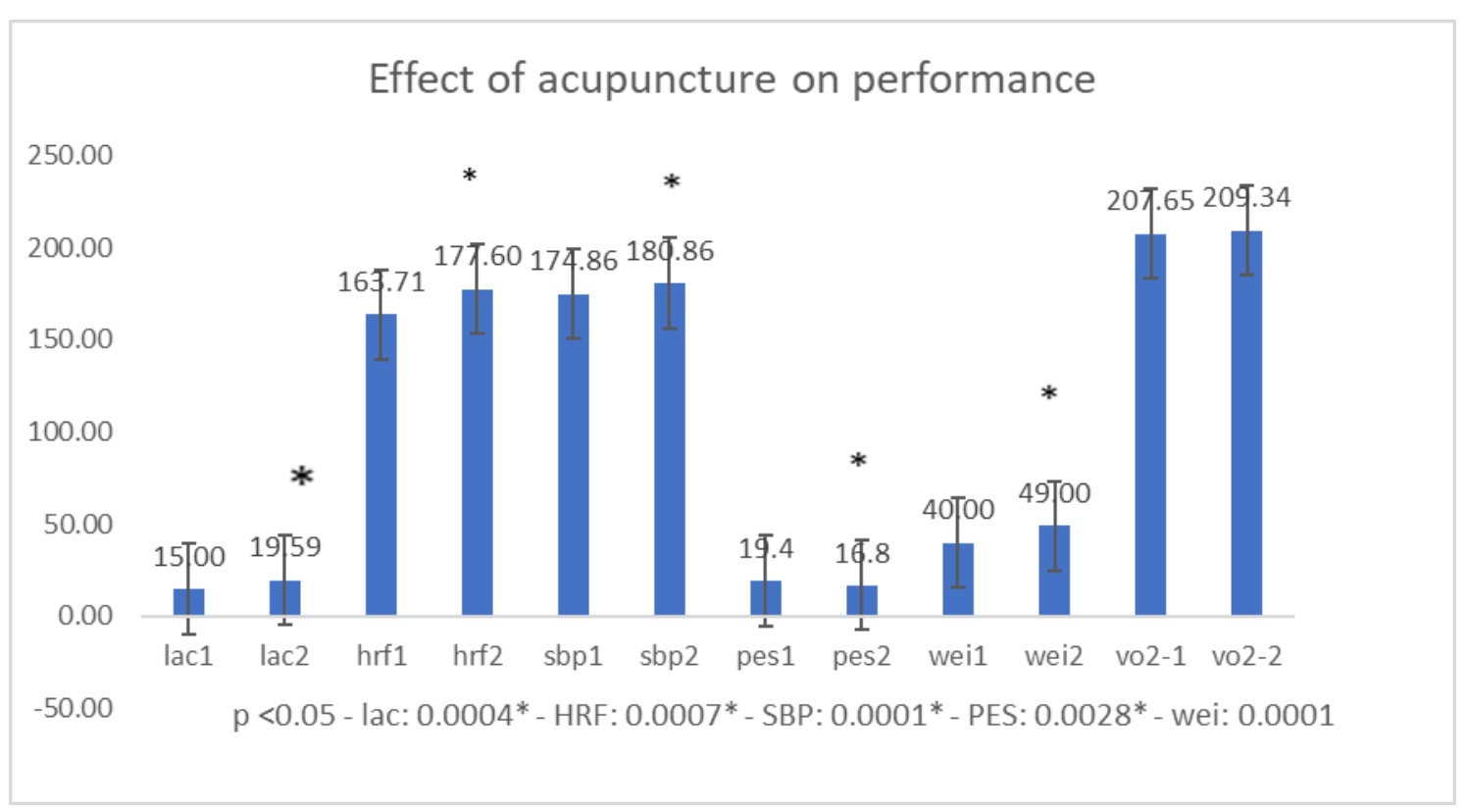

Legends: 1- final results without acupuncture; 2 -final results with acupuncture; lac - lactate $\mathrm{mM} / \mathrm{DL}$; HRF - heart rate frequency; SBP - systolic blood pressure; PES -perceived effort scale; WEI1 - weight without acupuncture (watts); WEI2 - weight with acupuncture (watts); VO2-1 - weight without acupuncture $\mathrm{ml} / \mathrm{kg} / \mathrm{min}^{-1}$; VO2-2 - weight without acupuncture $\mathrm{ml} / \mathrm{kg} / \mathrm{min}^{-1} ; \mathrm{x}$ - average; sd - standard deviation; Student $\mathrm{t}$ test; $\mathrm{p}$ value - Student $\mathrm{t}$ test; *statistical significance $\mathrm{p}<0.05$

\section{Discussion}

The aim of the present study was to determine the effect of acupuncture on lactate removal, heart rate recovery, systolic blood pressure and PES. In this respect, one 50-minute CrossFit session with 30-second recovery intervals every 3 minutes was held in order to change the physiological parameters investigated here without previous acupuncture and seven days after the technique. Acupuncture increased exertion, resulting in higher lactate concentration, heart rate and systolic blood pressure, while PES declined. Taken together, these data suggest that acupuncture may influence the subject's ability to perform physical work without perceiving this increased exertion.

A study conducted by Warr-Di Piero et al (21) aimed to determine lactate levels, heart rate and perceived exertion in athletes from different sports, submitted to high-intensity interval training. The authors observed an increase in the variables assessed during the activities and a positive correlation between lactate levels and heart rate similar to that observed here.

In a study with thirty individuals, with the experimental group submitted to manual acupuncture, another group to electroacupuncture and a control group that received no intervention, the manual and electroacupuncture groups displayed higher exercise intensity than the controls, corroborating the data found here $(11,22)$. The authors speculate that the 
pain threshold may have been changed in the aforementioned study, which could have led to higher levels of exertion without perception showing the same trend.

H. Ma, Liu, Wu, \& Zhang (12) observed that acupuncture at rest after sixteen 500-meter sprints at maximum velocity resulted in lower lactate concentrations in the acupuncture group compared to controls, in contrast to what occurred in the present study.

However, Kimura, Takeuchi, Yuri, \& (23) observed that acupuncture increased local circulation, primarily by producing nitric oxide, which induces vasodilation and stimulates the production of substances that alleviate pain. This was subsequently corroborated by Ma et al.(24), who reported that participants exhibited less perceived pain, allowing them to exercise with greater intensity, which may help explain our findings in the CrossFit group.

Applying needles at points PC6 and E36, the latter also used in the present study, was shown to reduce heart rate and lactate levels after 60 minutes (25), suggesting that the technique accelerated the recovery of athletes, similarly to that demonstrated here, where the physiological variables under study recovered in less time than those of the group submitted to acupuncture before exercise.

Applying one acupuncture session seems to maximize athletic exertion by decreasing pain perception, as previously reported by Hübscher, Vogt, Ziebart, \& Banzer(26).These authors concluded that a single session of manual acupuncture can significantly increase the level of isometric exertion in relation to controls.

\section{Conclusions}

Acupuncture seemed increased lactate accumulation, heart rate and blood pressure, suggesting that the exertion reached after acupuncture is higher than without acupuncture, and the decline in perceived exertion may have been due to the change in pain perception resulting from high-intensity physical activity during sports. The acupuncture technics will improve the athlete performance. On this case, we suggest use this practice thirty minutes before the work out or race. It will allow the effects of acupuncture during the activity. The limitations of this study were: the non-observations and quantification of the maximal oxygen consumption, the " $n$ " of the subjects relatively small, the impossibility of the quantification of the other biochemical markers that could interact with the markers here quantified, and, the food intake did not was controlled. Therefore, we suggest the observation of the female athletes, the control de food intake, a widest biochemical markers observation, to investigate other athletes of a high level of performance, insert and discuss other physical quality power, strength, stretching, and body composition.

\footnotetext{
Author Contributions: For research articles with several authors, a short paragraph specifying their individual contributions must be provided. The following statements should be used "Conceptualization, Carlos Soares Pernambuco and Rodrigo Gomes de Souza Vale; methodology, Rodrigo Gomes de Souza Vale.; formal analysis - Carlos Soares Pernambuco and Isabel Cristina Regazzi; , João Rafael Valentim-Silva; data curation Carlos Alberto de Souza Filho; Vinicius do Espirito Santo de Jesus.; writing-original draft preparation and writing-review and editing, João Rafael Valentim-Silva, Eliane da Silva Borges; Leandra Silva Cardoso; supervision, Estelio Henrique Martin Dantas. All authors have read and agreed to the published version of the manuscript.", please turn to the CRediT taxonomy for the term explanation. Authorship must be limited to those who have contributed substantially to the work reported.
} 
Acknowledgments: In this section you can acknowledge any support given which is not covered by the author contribution or funding sections. This may include administrative and technical support, or donations in kind (e.g., materials used for experiments).

Conflicts of Interest: Declare conflicts of interest or state "The authors declare no conflict of interest." Authors must identify and declare any personal circumstances or interest that may be perceived as inappropriately influencing the representation or interpretation of reported research results. Any role of the funders in the design of the study; in the collection, analyses or interpretation of data; in the writing of the manuscript, or in the decision to publish the results must be declared in this section. If there is no role, please state "The funders had no role in the design of the study; in the collection, analyses, or interpretation of data; in the writing of the manuscript, or in the decision to publish the results". 


\section{References}

1. Urroz P, Colagiuri B, Smith CA, Yeung A, Cheema BS. Effect of acupuncture and instruction on physiological recovery from maximal exercise: a balanced-placebo controlled trial. BMC Complement Altern Med. 2016;16:227.

2. Facey A, Dilworth L, Irving R. Adipocytic Contribution to Lactate Production in Male Athletes of West African Descent. The whole is more than the sum of its parts. 2014:57.

3. Brooks GA. Cell-cell and intracellular lactate shuttles. The Journal of physiology. 2009;587(23):5591-600.

4. Messonnier L, Freund H, Féasson L, Prieur F, Castells J, Denis C, et al. Blood lactate exchange and removal abilities after relative high-intensity exercise: effects of training in normoxia and hypoxia. European journal of applied physiology. 2001;84(5):403-12.

5. MacRae H, Dennis SC, Bosch AN, Noakes TD. Effects of training on lactate production and removal during progressive exercise in humans. Journal of applied physiology. 1992;72(5):1649-56.

6. Tomlin DL, Wenger HA. The relationship between aerobic fitness and recovery from high intensity intermittent exercise. Sports Medicine. 2001;31(1):1-11.

7. Kamali F, Sinaei E, Morovati M. Comparison of upper trapezius and infraspinatus myofascial trigger point therapy by dry needling in overhead athletes with unilateral shoulder impingement syndrome. Journal of sport rehabilitation. 2019;28(3):243-9.

8. Lima PR. Manual de Acupuntura. R. M. Trombeta E, editor. Porto Alegre, RS: Nova Letra; 2015.

9. PELHAM TW, HOLT LE, STALKER R. Acupuncture in Human Performance. The Journal of Strength \& Conditioning Research. 2001;15(2):266-71.

10. Ehrlich D, Haber P. Influence of acupuncture on physical performance capacity and haemodynamic parameters. International journal of sports medicine. 1992;13(06):486-91.

11. Dhillon S. The acute effect of acupuncture on 20-km cycling performance. Clinical Journal of Sport Medicine. 2008;18(1):76-80.

12. Ma H, Liu X, Wu Y, Zhang $N$. The intervention effects of acupuncture on fatigue induced by exhaustive physical exercises: a metabolomics investigation. Evidence-Based Complementary and Alternative Medicine. 2015;2015.

13. Ahmedov S. Ergogenic effect of acupuncture in sport and exercise: a brief review. The Journal of Strength \& Conditioning Research. 2010;24(5):1421-7.

14. Tang SK, Tse MMY, Leung SF, Fotis T. The effectiveness, suitability, and sustainability of non-pharmacological methods of managing pain in community-dwelling older adults: a systematic review. BMC public health. 2019;19(1):1488.

15. Scheirer MA. Is Sustainability Possible? A Review and Commentary on Empirical Studies of Program Sustainability. American Journal of Evaluation. 2005;26(3):320-47.

16. Prodanov CC, de Freitas EC. Metodologia do trabalho científico: métodos e técnicas da pesquisa e do trabalho acadêmico-2a Edição: Editora Feevale; 2013.

17. Pate RR, Pratt M, Blair SN, Haskell WL, Macera CA, Bouchard C, et al. Physical activity and public health: a recommendation from the Centers for Disease Control and Prevention and the American College of Sports Medicine. Jama. 1995;273(5):402-7. 
18. Borg G. Psychophysical scaling with applications in physical work and the perception of exertion. Scandinavian journal of work, environment \& health. 1990:55-8.

19. Hanson NJ, Martinez SC, Byl EN, Maceri RM, Miller MG. Increased rate of heat storage, and no performance benefits, with caffeine ingestion before a $10-\mathrm{km}$ run in hot, humid conditions. International journal of sports physiology and performance. 2018;14(2):196-202.

20. Silva R, Damasceno M, Cruz R, Silva-Cavalcante MD, Lima-Silva AE, Bishop DJ, et al. Effects of a 4-week high-intensity interval training on pacing during 5-km running trial. Brazilian journal of medical and biological research. 2017;50(12).

21. Warr-di Piero D, Valverde-Esteve T, Redondo-Castán JC, Pablos-Abella C, Sánchez-Alarcos Díaz-Pintado JV. Effects of work-interval duration and sport specificity on blood lactate concentration, heart rate and perceptual responses during high intensity interval training. PloS one. 2018;13(7):e0200690.

22. Kim S-B, Lee N-R, Park S-W, Ahn S-J, Heo H, Kim Y-H, et al. Electromagnetic acupuncture to enhance the effects of manual acupuncture on recovery from muscle fatigue of the quadriceps. Journal of acupuncture and meridian studies. 2014;7(5):250-7.

23. Kimura K, Takeuchi H, Yuri K, Wakayama I. Effects of nitric oxide synthase inhibition on cutaneous vasodilation in response to acupuncture stimulation in humans. Acupuncture in Medicine. 2013;31(1):74-80.

24. Ma S-x. Nitric oxide signaling molecules in acupoints: toward mechanisms of acupuncture. Chinese journal of integrative medicine. 2017;23(11):812-5.

25. Lin Z-P, Lan LW, He T-Y, Lin S-P, Lin J-G, Jang T-R, et al. Effects of acupuncture stimulation on recovery ability of male elite basketball athletes. The American journal of Chinese medicine. 2009;37(03):471-81.

26. Hübscher M, Vogt L, Ziebart T, Banzer W. Immediate effects of acupuncture on strength performance: a randomized, controlled crossover trial. European journal of applied physiology. 2010;110(2):353-8. 